\title{
Antimicrobial efficacy of herbal, homeopathic and conventional dentifrices against oral microflora: An in vitro study
}

\author{
Shivashankar Kengadaran ${ }^{1}$, Anusha Divvi ${ }^{1}$, Pradeep R. Kumar², Prakasam Gopinath ${ }^{3}$, Meignana I. Arumugham ${ }^{2}$, Sri D. Sakthi ${ }^{2}$
}

\author{
AFFILIATION \\ 1 Department of Public Heath Dentistry, Indira Gandhi Institute of Dental \\ Sciences, Sri Balaji Vidyapeeth, Pondicherry, India \\ 2 Department of Community Dentistry, Saveetha Dental College and \\ Hospitals, Chennai, India \\ 3 Department of Microbiology, Royal Dental College, Palakkad, Kerala, \\ India
}

CORRESPONDENCE TO

Shivashankar Kengadaran. Department of Public Heath Dentistry, Indira
Gandhi Institute of Dental Sciences, Sri Balaji Vidyapeeth, Pondicherry, 607402, India. E-mail: shiva.freee@gmail.com ORCID ID: https://orcid. org/0000-0002-6868-9639

KEYWORDS

fluoride, dentifrice, homeopathy, oral microflora

Received: 18 December 2019, Revised: 9 May 2020, Accepted: 17 May 2020

https://doi.org/10.18332/popmed/122529

\begin{abstract}
INTRODUCTION This study compares the efficacy of herbal, homeopathic, and conventional dentifrices, on oral microflora using antibiotic susceptibility tests.

METHODS Three strains of microorganisms, Streptococcus Mutans, Escherichia Coli, and Candida Albicans, were taken and incubated in Mutans media, Mueller Hilton agar, and Sabouraud Dextrose agar, respectively. Different dilutions $(1: 5,1: 10$ and $1: 15)$ of several brands of commercial toothpaste with different compositions were made. Sterile disks were incorporated with an equal amount of prepared toothpaste formulations using a micropipette. These disks were then placed equidistant to each other, and the plates were incubated for 24 hours.

RESULTS The zone of inhibition against $S$. Mutans is found to be higher in homeopathic dentifrice $24 \mathrm{~mm}, 19 \mathrm{~mm}$, and 20 $\mathrm{mm}$, followed by herbal dentifrice $19 \mathrm{~mm}, 17 \mathrm{~mm}$, and 13
\end{abstract}

$\mathrm{mm}$, and the least by conventional dentifrice $17 \mathrm{~mm}, 15 \mathrm{~mm}$ and no inhibition, at 1:5, 1:10 and 1:15 dilution, respectively. The zone of inhibition against $E$. Coli is found to be higher in herbal dentifrice $18 \mathrm{~mm}, 17 \mathrm{~mm}$ and $16 \mathrm{~mm}$ followed by conventional dentifrice $18 \mathrm{~mm}, 17 \mathrm{~mm}$, and $14 \mathrm{~mm}$, and no inhibition by homeopathic dentifrice at 1:5, 1:10 and 1:15 dilution, respectively. Zone of inhibition against $C$. Albicans is found to be higher in herbal dentifrice $14 \mathrm{~mm}, 12 \mathrm{~mm}$ and $9 \mathrm{~mm}$ followed by conventional dentifrice $14 \mathrm{~mm}, 9 \mathrm{~mm}$, and no inhibition, and the least by homeopathic dentifrice $10 \mathrm{~mm}, 9 \mathrm{~mm}$ and $7 \mathrm{~mm}$, at 1:5, 1:10 and 1:15 dilutions, respectively.

CONCLUSIONS Toothpaste formulations containing homeopathic and natural antimicrobial agents were more effective in controlling the oral microflora compared to toothpaste containing synthetic antimicrobial agents like triclosan.

\section{INTRODUCTION}

Globally, dental caries and gingivitis are the most common oral diseases that affect people of all ages ${ }^{1}$. The occurrence of dental caries is approximately $60-65 \%$ among the Indian population ${ }^{2,3}$. Dental diseases are primarily caused by the virulence of complex oral micro-communities ${ }^{4}$. Dental plaque micro-organisms degrade the dietary carbohydrates and produce lactic acid, which leads to localized demineralization and dental caries, eventually, ${ }^{5,6}$.

Poor oral hygiene is the major risk factor for the accumulation of microbes and their harmful activities ${ }^{4,7}$.
Most of the dental diseases can be prevented from occurring by the simple practice of personal hygiene habits ${ }^{8,9}$. Besides mechanical cleansing of teeth, the use of chemical agents has been proposed as an effective method of reducing plaquemediated diseas $\mathrm{e}^{10}$. Most effective among them is the tooth brushing habit as the antibacterial efficacy of the toothpaste has a major role to play in the outcome $\mathrm{e}^{11,12}$.

Triclosan, an antibacterial agent, which is a common ingredient in toothpaste, helps to prevent gingivitis by reducing calculus formation ${ }^{13}$. Herbal products contain antimicrobial agents and may be more appealing as they 
do not require alcohol, preservatives, and flavours, for their activity ${ }^{14,15}$. The basic homeopathic principle is that a substance in fewer doses efficiently cures those similar symptoms that would require a larger dose. It has been proven that the homeopathic formulations are non-toxic, antiinflammatory, and antimicrobial ${ }^{16}$. Considering the availability of abundant brands of toothpaste in the market, their efficacy to control the bacterial count has to be monitored and analysed scientifically. Currently, the increased popularity of herbal products has mandated that dental professionals evaluate the effectiveness of these products in the prevention of oral ailments and provide evidence-based suggestions to their patients for making a better choice ${ }^{17}$. Also, there is a growing interest around the world to study the benefits of various medicinal plants, over the last decade ${ }^{18}$.

Antibiotic susceptibility testing is the method employed for testing the effectiveness of agents against specific microorganisms ${ }^{19}$. Disk diffusion assay offers many advantages such as simplicity, low cost, ability to test large numbers of microorganisms and antimicrobial agents, and the ease to interpret results ${ }^{19}$. Hence, this study aims to compare the efficacy of herbal, homeopathic, and conventional dentifrices, on oral microflora using antibiotic susceptibility tests.

\section{METHODS}

For the present in vitro study, three strains of microorganisms, Streptococcus Mutans, Escherichia Coli, and Candida Albicans, were considered. The media used were Mutans media, Mueller Hilton agar, and Sabouraud Dextrose agar, respectively, for each micro-organism. We prepared dilutions of several brands of commercial toothpaste with different compositions. Double distilled water, pyrogen-free water, test tubes, Petri plates, micropipettes, and gel puncher were the other materials utilized for the study.

Solutions of selected dentifrices were made by mixing $1 \mathrm{~g}$ of dentifrices in $4 \mathrm{~mL}$ of distilled water to give 1:5 dilutions, in a sterile container. Further dilutions were made by mixing $1 \mathrm{~g}$ of toothpaste with $9 \mathrm{~mL}$ and $14 \mathrm{~mL}$ to give $1: 10$ and 1:15 dilutions, respectively (Figure 1).

Three strains of microorganisms, S. Mutans, E. Coli, and C. Albicans, were grown in Mutans media, Mueller Hilton agar, and Sabouraud Dextrose agar media, respectively. These bacteria were inoculated in their respective medium by the swab method (Figure 2).

Sterile disks were incorporated with an equal amount of different dilutions of prepared toothpaste formulations using a micropipette. These disks were then placed equidistant to each other and the plates were incubated for 24 hours. The zones of inhibition were measured to the nearest whole $\mathrm{mm}$ using a graduated ruler by holding the test plates in front of a desk lamp.

Ethical clearance was obtained from the institutional ethical committee before beginning the study.

\section{RESULTS}

Zone of inhibition against S. Mutans was found to be higher in homeopathic dentifrice followed by herbal dentifrice, and the least by conventional dentifrice, and no inhibition at 1:5, 1:10 and 1:15 dilutions, respectively (Table 1). Zone of inhibition against $C$. Albicans was found to be higher in herbal dentifrice followed by conventional dentifrice, and no inhibition, and the least by homeopathic dentifrice at 1:5, 1:10 and 1:15 dilutions, respectively. Zone of inhibition against $E$. Coli was found to be higher in herbal dentifrice followed by conventional dentifrice, and no inhibition by homeopathic dentifrice at 1:5, 1:10 and 1:15 dilutions, respectively.

Figure 1. Preparation of dentifrice formulation
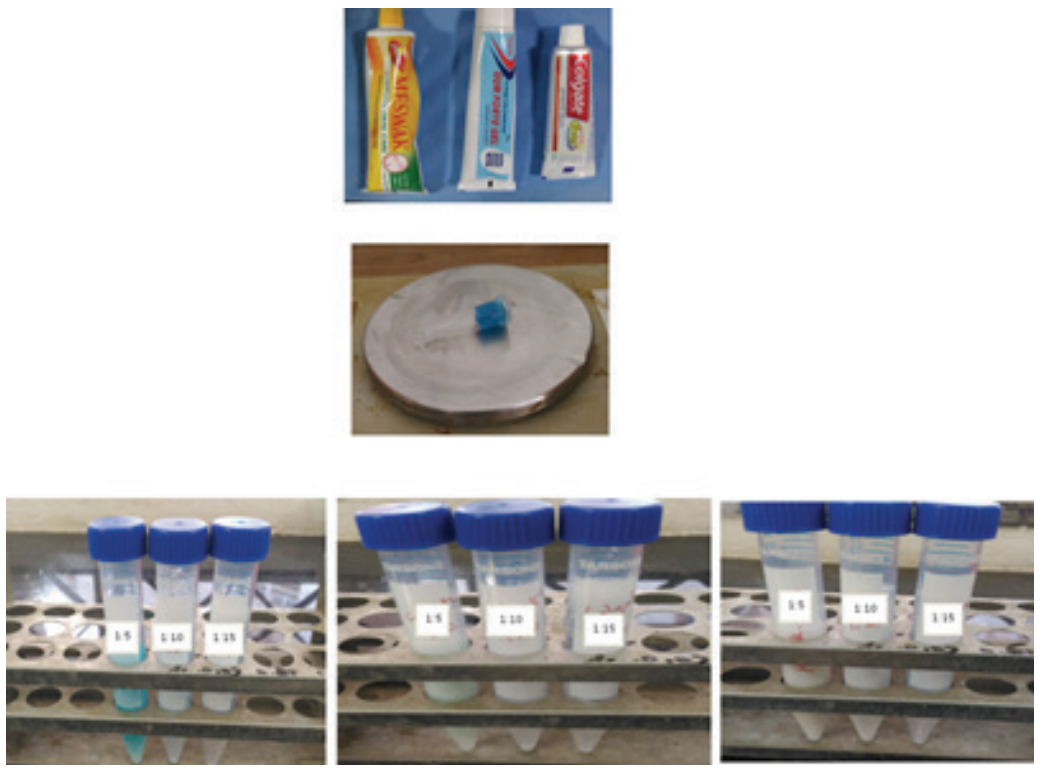
Figure 2. Antimicrobial activity of various dentifrices against various microorganisms

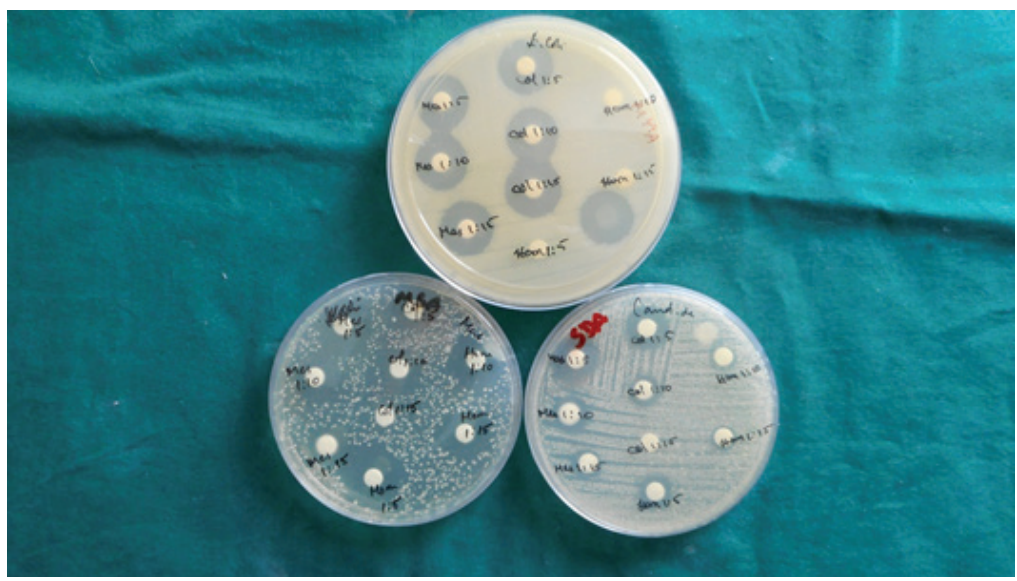

The zone of inhibition is calculated as the diameter of the area around the disk that is free of microbial colonies.

Table 1. Zone of inhibition ( $\mathrm{mm}$ ) of various types of dentifrices at three dilutions

\begin{tabular}{|l|r|r|r|}
\hline \multirow{2}{*}{$\begin{array}{l}\text { Dentifrices } \\
\text { Streptococcus Mutans }\end{array}$} & \multicolumn{3}{|c|}{ Zone of inhibition $(\mathbf{m m})$} \\
\cline { 2 - 4 } Herbal dentifrice & 19 & 17 & $1: 15$ \\
\hline Homeopathic dentifrice & 24 & 19 & 13 \\
Conventional dentifrice & 17 & 15 & 0 \\
\hline Candida Albicans & & & \\
\hline Herbal dentifrice & 14 & 12 & 9 \\
Homeopathic dentifrice & 10 & 9 & 7 \\
Conventional dentifrice & 14 & 9 & 0 \\
\hline E. Coli & & & \\
\hline Herbal dentifrice & 18 & 17 & 16 \\
Homeopathic dentifrice & 0 & 0 & 0 \\
Conventional dentifrice & 18 & 17 & 14
\end{tabular}

\section{DISCUSSION}

Microbial biofilms are the major causative factors of caries and periodontal disease, and it is of utmost importance to control these biofilms by mechanical and chemical debridement ${ }^{15}$. Prevalence of dentinal caries result from poor oral hygiene. It has been proven scientifically that maintaining good oral hygiene is the key to preventing dental diseases ${ }^{4}$. Toothpaste is a gel dentifrice used with a toothbrush accessory to clean and maintain oral hygiene.

There is a paucity of literature about the efficacy of different types of dentifrices against oral microorganisms. Hence, this study was conducted to test the antimicrobial efficacy of three different dentifrices (herbal, homeopathic, and conventional fluoride containing) against S. Mutans, E.
Coli, and C. Albicans.

Three different dentifrice formulations 1:5, 1:10, and $1: 15$, were made by mixing distilled water with dentifrice. The media used were Mutans media, Mueller Hilton agar, and Sabouraud Dextrose agar media, respectively, for each microorganism by swab method. The antimicrobial efficacy was then tested using the disk diffusion method.

In this study, the antimicrobial efficacy of dentifrice formulations against $S$. Mutans was measured as a zone of inhibition. The zone of inhibition is found to be higher in homeopathic dentifrice $24 \mathrm{~mm}, 19 \mathrm{~mm}$, and $20 \mathrm{~mm}$, followed by herbal dentifrice $19 \mathrm{~mm}, 17 \mathrm{~mm}$, and $13 \mathrm{~mm}$, and the least by conventional dentifrice $17 \mathrm{~mm}, 15 \mathrm{~mm}$, and no inhibition, at 1:5, 1:10 and 1:15 dilutions, respectively. Similar results were obtained in the study conducted by Mohankumar et al. ${ }^{17}$ where herbal toothpaste was found to have similar antibacterial activity compared to the conventional toothpaste. The herbal toothpaste with a combination of different varieties of herbs has better antimicrobial activity compared to those with a single ingredient. However, studies conducted by Anushree et al. ${ }^{14}$ and Prasanth ${ }^{3}$ showed fluoride and triclosan containing dentifrices had higher zones of inhibition at higher dilutions. Homeopathicbased dentifrices showed antimicrobial efficacy against $S$. Mutans since the major ingredients of its formulations were kreosotum, Plantago major, and calendula ${ }^{14}$.

Similar results were observed by Gibraiel et al. ${ }^{12}$ in their study where the toothpaste having natural formulation had given maximum zones of inhibition against $E$. Coli at 1:1 dilution. However, studies conducted by Anushree et al. ${ }^{14}$ and Prasanth ${ }^{3}$ showed fluoride and triclosan containing dentifrices had higher zones of inhibition at higher dilutions. The difference in the result may be attributed to the active ingredient used in the herbal dentifrices. However, studies conducted by Anushree et al. ${ }^{14}$ and Prasanth ${ }^{3}$ showed fluoride and triclosan containing dentifrices had higher 
zones of inhibition at higher dilutions. This difference may be because different herbal products exhibit different levels of antimicrobial activity.

Herbal dentifrice showed an increased antimicrobial activity than homeopathic and conventional dentifrices, against all the test organisms. It is thus necessary to acquire and preserve this traditional system of medicine by proper documentation. Further, in vivo studies have to be conducted to assess the antimicrobial effectiveness of these dentifrices.

\section{CONCLUSIONS}

The results of the present study show that toothpaste formulations containing homeopathic and natural antimicrobial agents were more effective in controlling oral microflora compared to conventional toothpaste. This provides insight to the dentists that homeopathic and herbal dentifrices are effective in controlling microorganisms related to major oral diseases and can be advised to the patients.

\section{REFERENCES}

1. Saini S, Aparna, Gupta N, Mahajan A, Arora D R. Microbial flora in orodental infections. Indian J Med Microbiol. 2003;21(2):111-114. PMID:17642993.

2. Ramchandran K, Rajan BP, Shanmungan S. Epidemiological studies of dental disorders in Tamilnadu population, prevalence of dental caries and periodontal disease. J Indian Dent Assoc. 1973;45(4):65-70. PMID:4514703.

3. Prasanth M. Antimicrobial Efficacy of Different Toothpastes and Mouthrinses: An In Vitro Study. Dent Res J. 2011;8(2):8594. PMID:22013468.

4. Namita P, Mukesh R, Vijay K. Camellia sinensis (Green tea): A review. Glob J Pharmacol. 2012;6:52-59. doi:10.1002/0471701343.sdp30621

5. Taylor PW, Hamilton Miller JM, Stapleton PD. Antimicrobial properties of green tea catechins. Food Sci Technol Bull. 2005;2(7):71-81. doi:10.1616/1476-2137.14184

6. Smiline GAS, Pandi SK, Hariprasad P, Raguraman R. A preliminary study on the screening of emerging drug resistance among the caries pathogens isolated from carious dentine. Indian J Dent Res. 2012;23(1):26-30. doi:10.4103/0970-9290.99033

7. Balakrishnan M, Simmonds RS, Tagg JR. Dental caries is a preventable infectious disease. Aust Dent. 2000;45(4):235245. doi:10.1111/j.1834-7819.2000.tb00257.x

8. Control and prevention of Dental Caries. 3. Quintessence international. In: Cariology. Chicago, IL: Quintessence Publishing Company; 1989. p. 359-360.

9. Priya S, Uma E, Reddy MKR. Effect of different types of tea on Streptococcus mutans: An in vitro study. Indian J Dent Res.

\section{CONFLICTS OF INTEREST}

The authors have completed and submitted the ICMJE Form for Disclosure of Potential Conflicts of Interest and none was reported.

\section{2;23(1):43-48. doi:10.4103/0970-9290.99037}

10. Menendez A, Li F, Michalek SM, Kirk K, Makhija SK, Childers NK. Comparative analysis of the antibacterial effects of combined mouth rinses on Streptococcus mutans. Oral Microbiol Immunol. 2005;20(1):31-34. doi:10.1111/j.1399-302x.2004.00189.x

11. Fabiana O, Claudio Mendes Pannuti, Ana Vitoria Imbronito, Wellington P, Luciana S, Nivea Maria de Freitas. Efficacy of a herbal toothpaste on patients with established gingivitis - a randomized controlled trial. Braz Oral Res. 2006;20(2):172177. doi:10.1590/s1806-83242006000200015

12. Gibraiel F, Rajput M, Singh MR, Singh M, Saxena N, Vishal A, Jha KA. In Vitro Study to Investigate the Antimicrobial Efficacy of Different Toothpastes and Mouth Rinses. RJPBCS. 2014;5(2):245. https://www.rjpbcs.com/pdf/2014_5(2)/ [27].pdf. Accessed May 9, 2020.

13.Shubhra V, Dakshi A, Vidya D, Hari P. Comparative Evaluation of $0.2 \%$ Chlorhexidine Versus Herbal Oral Rinse on Plaque Induced Gingivitis. J Indian Ass Pub Health Dent. 2013;19(10):55-62. http://www.jiaphd.org/article. asp?issn=2319-5932;year=2012; volume=10;issue=19; ;pag e=55; epage=62; aulast=Vaish;type=0. Accessed May 9, 2020.

14. Anushree B, Fawaz MA, Narahar R, Shahela T, Syed A. Comparison of Antimicrobial Efficacy of TriclosanContaining, Herbal and Homeopathy Toothpastes- An Invitro Study. J Clin Diagn Res. 2015;9(10):DC05-08. doi:10.7860/JCDR/2015/11984.6626

15. Sean SL, WU Zhang, Yiming L. The antimicrobial potential of 14 natural Herbal dentifrices: Results of an in vitro diffusion method study. J Am Dent Assoc. 2004;135(8):1133-1141. doi:10.14219/jada.archive.2004.0372

16. Bhat SS, Sargod SS, George D. Dentistry and homeopathy: an overview. Dent Update. 2005;32(8):486-491. doi:10.12968/denu.2005.32.8.486

17. Mohankumar KP, Priya NK, Madhushankari GS. AntiCariogenic Efficacy of Herbal and Conventional Tooth Pastes-A Comparative In-Vitro Study. J Int Oral Health. 2013;5(2):8-13. PMID:24155585.

18. Deshpande RR, Kachare PR, Sharangpani GA, Varghese VK, Bahulkar SS. Comparative evaluation of antimicrobial efficacy of two commercially available dentifrices (fluoridated and herbal) against salivary microflora. Int J Pharm Pharm Sci. 2014;6(6):72-74. https://pdfs.semanticscholar.org/adc7/ b4478677cfac83bfce6abd83f79b75f6d8d6.pdf. Accessed May 9, 2020.

19. Balouiri M, Sadiki M, Ibnsouda S. Methods for in vitro evaluating antimicrobial activity: A review. J Pharm Anal. 2016;6(2):71-79. doi:10.1016/j.jpha.2015.11.005

PROVENANCE AND PEER REVIEW

Not commissioned; externally peer reviewed. 